\title{
(6) OPEN ACCESS \\ Mixed ethnicity and behavioural problems in the Millennium Cohort Study
}

\author{
Afshin Zilanawala, Amanda Sacker, Yvonne Kelly
}

- Additional material is published online only. To view please visit the journal online (http://dx.doi.org/10.1136/ archdischild-2015-309701).

Department of Epidemiology and Public Health, University College London, London, UK

\section{Correspondence to}

Dr Afshin Zilanawala,

Department of Epidemiology and Public Health, University College London, 1-19 Torrington Place, London WC1E 6BT, UK; afshin.zilanawala@ucl.ac.uk

Received 4 September 2015 Revised 26 January 2016 Accepted 29 January 2016 Published Online First 24 February 2016

\begin{abstract}
Background The population of mixed ethnicity individuals in the UK is growing. Despite this demographic trend, little is known about mixed ethnicity children and their problem behaviours. We examine trajectories of behavioural problems among non-mixed and mixed ethnicity children from early to middle childhood using nationally representative cohort data in the UK.

Methods Data from 16330 children from the Millennium Cohort Study with total difficulties scores were analysed. We estimated trajectories of behavioural problems by mixed ethnicity using growth curve models. Results White mixed (mean total difficulties score: 8.3), Indian mixed (7.7), Pakistani mixed (8.9) and Bangladeshi mixed (7.2) children had fewer problem behaviours than their non-mixed counterparts at age 3 (9.4, 10.1, 13.1 and 11.9, respectively). White mixed, Pakistani mixed and Bangladeshi mixed children had growth trajectories in problem behaviours significantly different from that of their non-mixed counterparts.

Conclusions Using a detailed mixed ethnic classification revealed diverging trajectories between some non-mixed and mixed children across the early life course. Future studies should investigate the mechanisms, which may influence increasing behavioural problems in mixed ethnicity children.
\end{abstract}

\section{INTRODUCTION}

Existing evidence shows ethnic variation for a variety of markers of health across the early life course. ${ }^{1-3}$ Ethnic inequalities may be a consequence of group characteristics or due to social/economic disadvantage. ${ }^{4}$ Very little research examines the consequences of mixed ethnicity status for children. ${ }^{5}$ Research on mixed ethnic differences in child behaviour is limited due to broad or homogenous groups in data. Investigating children's problem behaviours during the early life course is relevant because behavioural problems have been linked to subsequent academic achievement, adult economic well-being and crime. ${ }^{67}$

Only one study investigated mixed ethnic differences in behavioural problems during early childhood and reported no link between mixed ethnicity and behavioural problems among 3-year-old children. ${ }^{5}$ Although evidence was based on a nationally representative cohort, a single, heterogeneous 'mixed' category was used. A London-based study (DASH) examining 11-13-year-old children found no difference in problem behaviours between mixed black Caribbean/white and their white counterparts. ${ }^{8}$ Both studies use a cross-sectional design, making it difficult to discern whether and how early

\section{What is already known on this topic}

- Research has documented ethnic inequalities in health across the early life course.

- There is a paucity of longitudinal research investigating mixed ethnic differences in children's behavioural problems using a comprehensive mixed ethnic classification.

\section{What this study adds}

- We find White mixed, Indian mixed, Pakistani mixed and Bangladeshi mixed 3-year-old children to have fewer problem behaviours than their non-mixed counterparts.

- Growth curve models demonstrated that White mixed, Pakistani mixed and Bangladeshi mixed children experienced increases in problem behaviours compared with their non-mixed counterparts, particularly after age 7 .

potential differentials in behavioural problems emerge. Another paper using DASH data looked at growth trajectories to examine problem behaviours showed more favourable child behaviour profiles among adolescent (11-16 year olds) ethnic minority groups in the UK; $;^{9}$ however, authors did not disaggregate ethnic minority groups by mixed status.

Research has called for more robust evidence on mixed ethnicity children's behavioural problems. ${ }^{8}$ Our analysis extends prior research in several ways. First, using a nationally representative cohort study, we estimate trajectories in behavioural problems from age 3 to 11 , allowing us to detect declines or inclines in problem behaviours. Second, we use a detailed mixed ethnic classification, disaggregating ethnic groups accounts for the heterogeneity in socioeconomic, migratory and health profiles of these groups. ${ }^{10}$ Third, we compare the behavioural problems' trajectories of mixed ethnicity cohort members with those of their non-mixed counterparts. This approach allows us to clarify whether children's problem behaviours are sensitive to their mixed ethnic status.

\section{METHODS}

Data

The Millennium Cohort Study (MCS) is a nationally representative longitudinal study of 18552 infants born in the UK between September 2000 
and January 2002. ${ }^{11}$ The sample was clustered at the electoral ward (an administrative unit level) such that disadvantaged residential areas and areas with a high proportion of ethnic minority residents are over-represented. The main respondents are primarily mothers. The first interview was when cohort members were 9 months of age, and follow-up sweeps were conducted at ages 3, 5, 7 and 11 years. During interviews, the main respondent was asked about the cohort members' behavioural problems.

Problem behaviours were assessed using the Strengths and Difficulties Questionnaire (SDQ), ${ }^{12}$ which was completed by the main respondent when cohort members were approximately 3 , 5, 7 and 11 years old. We used the total difficulties score, which is the sum of four behavioural domains (peer problems, conduct disorders, hyperactivity and emotional problems) and ranges from 0 to 40 (a higher score indicates more problem behaviours). A one-point change on the SDQ is clinically meaningful. ${ }^{13}{ }^{14}$ The SDQ has been validated in ethnically diverse populations. ${ }^{15}$

Ethnic categories were constructed using mother's reports of the cohort child's ethnicity and were based on census categories. We drew upon father's ethnicity to facilitate the ethnic classification of the cohort child. A cohort child's ethnicity is categorised as mixed if the main respondent (usually the mother) chose a mixed category or if the ethnic categories for the child's parents were different. If the main respondent chose a 'mixed' ethnic category for the child, but the categories of the child's natural parents were the same, we reclassified the child according to the parents' ethnicity. Ethnic categories used for analysis are white; Indian; Pakistani; Bangladeshi; black Caribbean; black African; other and their 'mixed' counterparts. The white mixed group is made up of children whose parents are from different white backgrounds, for example, a white mixed child might have a white European mother and a white British father.

Our analyses included cohort member's gender, age, sweep at assessment as a measure of time and a quadratic term for time. The linear but not the quadratic term varied by ethnicity. We explored the impact of equivalised household income in quintiles, measured as a time-varying variable (middle-income quintile is reference) ${ }^{16}$ in sensitivity analyses.

Behavioural outcomes are moderated by multiple births, ${ }^{17}$ and therefore, we analysed data on singleton born children. We excluded from our analysis children who were reported to have attention-deficit/hyperactivity disorder, autism or Asperger's syndrome. The sample was restricted to children for whom a parent report of problem behaviours was available and for whom ethnicity was observed. For descriptive results, we estimated means on problem behaviours using cross-sectional samples. For the growth curve analyses, we pooled data from ages $3,5,7$ and 11 , allowing each child to contribute more than one observation. The pooled analyses had 16330 children and a total of 51509 child-years.

\section{Statistical analysis}

We estimated growth curve models for children's behavioural problems trajectories for the analytic sample. ${ }^{18}$ We estimated a behavioural problems intercept and slope for each cohort member across time (level 1), regressed the mean intercept and slope on each cohort member's ethnicity (level 2), and estimated the residual variance in the intercept and slope. We present figures illustrating initial problem behaviours at age 3 and trajectories in problem behaviours by non-mixed and mixed ethnic groups. All analyses are weighted to adjust for non-response and the unequal probability of being sampled.

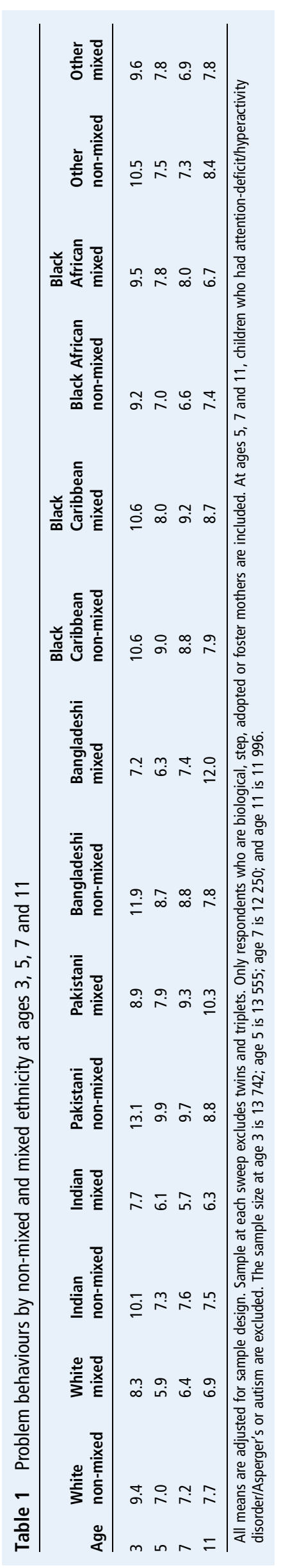



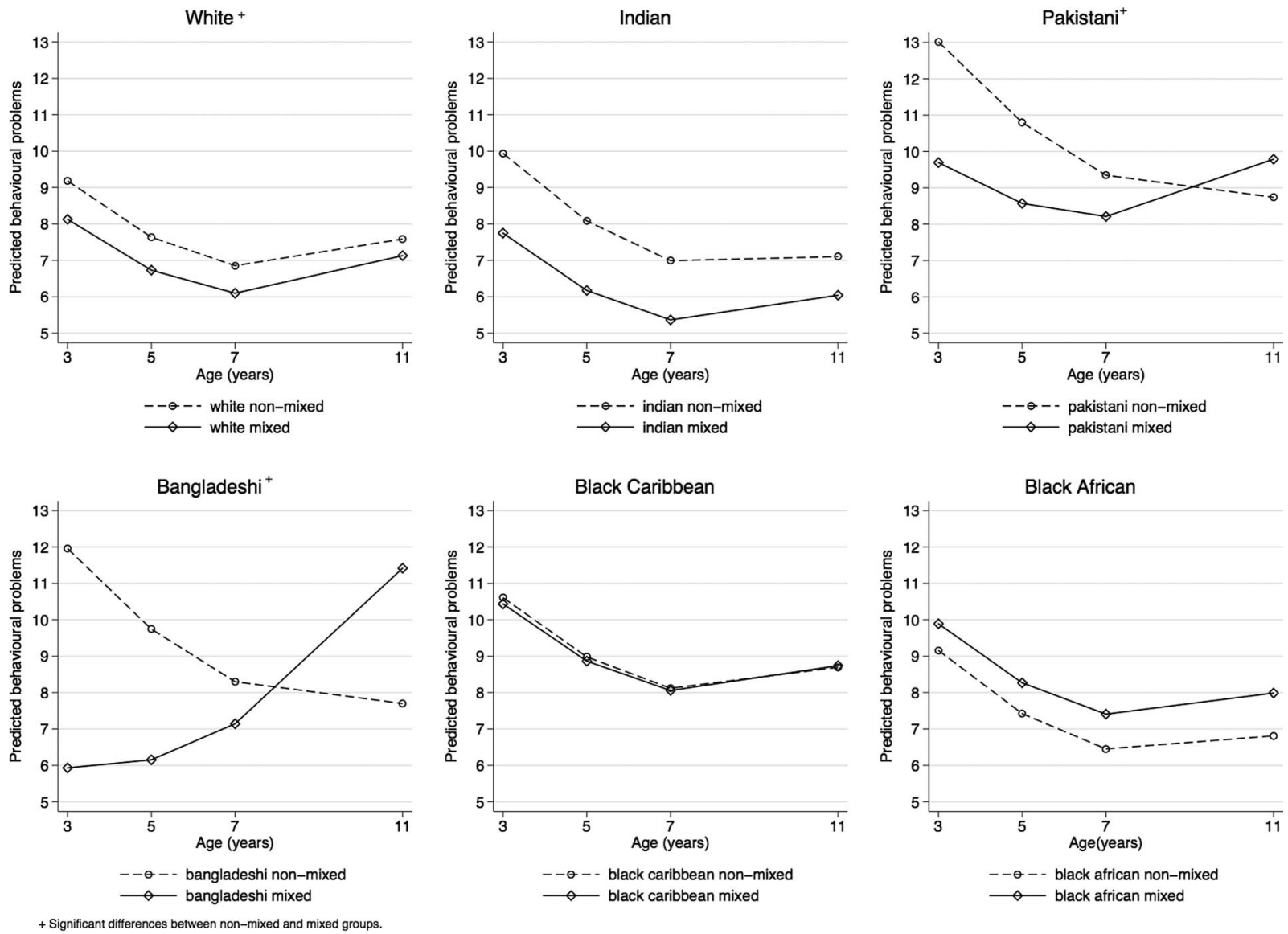

Figure 1 Predicted behavioural problems by mixed ethnicity.

\section{RESULTS}

\section{Mixed ethnicity and children's problem behaviours}

Table 1 presents mean problem behaviours by non-mixed and mixed ethnicity across the early life course. All sample sizes are reported in online supplementary appendix table 2. At age 3, most mixed ethnicity children had fewer problem behaviours compared with their non-mixed counterparts; White mixed, Indian mixed, Pakistani mixed and Bangladeshi mixed children had fewer problem behaviours than their non-mixed counterparts. Black Caribbean non-mixed and mixed children did not differ in their mean scores. Black African mixed children had slightly more problem behaviours compared with their nonmixed counterparts, but means were not statistically different (9.5 vs 9.2 , respectively).

The patterns in problem behaviours are less consistent at age 11. White and Indian mixed children have fewer problem behaviours compared with their non-mixed counterparts. Pakistani, Bangladeshi and Black Caribbean mixed children have more problem behaviours than their non-mixed counterparts. Black African mixed children have fewer behavioural problems than their non-mixed counterparts. In order to closely scrutinise the increase and decrease in problem behaviours over time, we turn to growth curve models to more accurately distinguish differences between mixed ethnicity children and their counterparts.

\section{Growth trajectories}

Figure 1 displays the average fitted growth trajectories in problem behaviours by mixed status conditional on age and gender. Regression coefficients are in online supplementary appendix table 1 (model 1). The rate of growth in problem behaviours for white mixed children is significantly different from their non-mixed counterparts $(b=0.075$, se $=0.037)$. Indian children experienced a decline in problem behaviours over time and trajectories do not differ between non-mixed and mixed Indian children. Pakistani and Bangladeshi mixed children experience rates of change in problem behaviours that are significantly different from those of their non-mixed counterparts. There were no differences in the rates of change between non-mixed and mixed black Caribbean children. Lastly, black African non-mixed children experienced a decline in problem behaviours but their trajectories did not significantly differ from their mixed counterparts.

In sensitivity analyses, adjustments for equivalised household income decreased the mean differences in intercepts of child behaviour between mixed and non-mixed ethnic minorities. However, the magnitude and significance of trajectories were not sensitive to adjustments for income (see online supplementary appendix table 1 , model 2 ).

\section{DISCUSSION}

In this paper, we extend our understanding of mixed ethnicity differences in behavioural problems by examining children's trajectories beginning in early childhood and including a comprehensive mixed ethnic classification using nationally representative data. At age 3, we find white mixed, Indian mixed, Pakistani mixed and Bangladeshi mixed children to have fewer problem behaviours than their non-mixed counterparts. This finding is in stark contrast to previous research finding no differences in problem behaviours between a catch-all mixed group and nonmixed 3-year-old children. ${ }^{5}$ Growth curve models show that white 
mixed, Pakistani mixed and Bangladeshi mixed children experienced increases in problem behaviours compared with their nonmixed counterparts, most notably after age 7 .

Our findings suggest that by age 11 some mixed children have more problem behaviours than their non-mixed counterparts. There are two possible explanations for this result. One, mixed ethnicity as a category may reflect not only social stratification processes but also shifts in identity and potential confusions between personal and social identities. ${ }^{19}$ The behavioural problems among mixed 11-year-old children in our data may very well reflect the strain from the nuanced nature of forming and maintaining a mixed ethnicity heritage. Second, we do not observe differences in problem behaviours between non-mixed and mixed black Caribbean children, but such differences are evident between non-mixed and mixed statuses among Pakistani and Bangladeshi children. This could be explained by the normative nature of interethnic partnerships among the black Caribbean community, while mixed partnerships are less common in South Asian communities. ${ }^{5}$ It could be that mixed partnerships yield less anxiety and strain, factors affecting child outcomes, when such interethnic families are more common within an ethnic group. Future studies should disentangle the influence of family context, psychosocial factors and problem behaviours among mixed ethnicity children.

The aim of this short report was to describe, for the first time, trajectories in problem behaviours for non-mixed and mixed ethnicity children in a nationally representative sample. White mixed, Pakistani mixed and Bangladeshi mixed children had significantly different growth trajectories compared with their non-mixed counterparts. Future research might examine the mechanisms, such as school, psychosocial and sociodemographic factors, behind these differences.

Acknowledgements The authors thank the MCS families for their time and cooperation, as well as the MCS team at the Institute of Education.

Contributors All authors approved the final version of the article and have participated sufficiently in the work to take public responsibility for appropriate portions of the content. Specific author contributions as follows: YK generated the idea for the paper. AZ analysed the data based on a plan developed with YK and AS. AZ wrote the manuscript with YK and AS providing comments.

Funding The authors would also like to acknowledge the funding for this work from the UK Economic and Social Research Council RES 177-25-0012.

Competing interests None declared.

Patient consent Obtained.

Ethics approval Ethical approval for the MCS was gained from the relevant ethics committees.

Provenance and peer review Not commissioned; externally peer reviewed.
Open Access This is an Open Access article distributed in accordance with the terms of the Creative Commons Attribution (CC BY 4.0) license, which permits others to distribute, remix, adapt and build upon this work, for commercial use, provided the original work is properly cited. See: http://creativecommons.org/ licenses/by/4.0/

\section{REFERENCES}

1 Maynard M, Harding S. Perceived parenting and psychological well-being in UK ethnic minority adolescents. Child Care Health Dev 2010;36:630-8.

2 Zilanawala A, Sacker A, Nazroo J, et al. Ethnic differences in children's socioemotional difficulties: findings from the Millennium Cohort Study. Soc Sci Med 2015;134:95-106.

3 Zilanawala A, Davis-Kean P, Nazroo J, et al. Race/ethnic disparities in early childhood BMI, obesity and overweight in the United Kingdom and United States. Int J Obes (Lond) 2015;39:520-9.

4 Nazroo J, Jackson J, Karlsen S, et al. The Black diaspora and health inequalities in the US and England: does where you go and how you get there make a difference? Sociol Health IIIn 2007;29:811-30.

5 Platt L. How do children of mixed partnerships fare in the United Kingdom? Understanding the implications for children of parental ethnic homogamy and heterogamy. Ann Am Acad Political Soc Sci 2012;643:239-66.

6 Duncan GJ, Dowsett CJ, Claessens A, et al. School readiness and later achievement. Dev Psychol 2007:43:1428.

7 Cunha F, Heckman JJ. The economics and psychology of inequality and human development. J Eur Econ Assoc 2009;7:320-64

8 Maynard MJ, Harding S, Minnis H. Psychological well-being in Black Caribbean, Black African, and white adolescents in the UK Medical Research Council DASH study. Soc Psychiatry Psychiatr Epidemiol 2007:42:759-69.

9 Astell-Burt T, Maynard MJ, Lenguerrand E, et al. Racism, ethnic density and psychological well-being through adolescence: evidence from the determinants of adolescent social well-being and health longitudinal study. Ethn Health 2012;17:71-87

10 Modood T. Ethnic differentials in educational performance. In: Mason D, ed. Explaining ethnic differences: changing patterns of disadvantage in Britain. Bristol: The Policy Press, 2003:53-68.

11 Plewis I, Calderwood L, Hawkes D, et al. Millennium cohort study: technical report on sampling. London: Institute of Education, University of London, 2004;16.

12 Goodman R. Psychometric properties of the strengths and difficulties questionnaire. J Am Acad Child Adolesc Psychiatry 2001;40:1337-45.

13 Cohen J. Statistical power analysis for the behavioral sciences. Lawrence Erlbaum Associates, Inc, 1977.

14 Goodman A, Goodman R. Strengths and difficulties questionnaire as a dimensional measure of child mental health. J Am Acad Child Adolesc Psychiatry 2009;48:400-3.

15 Meltzer H, Gatward R, Goodman R, et al. Mental health of children and adolescents in Great Britain. TSO London, 2000.

16 Calderwood EJ, MacDonald J, Shepherd P, et al. Millennium Cohort Study First, Second and Third Surveys A Guide to the Datasets. 2008.

17 Hansen K, Joshi H. Millennium Cohort Study Third Survey: a user's guide to initial findings: Centre for Longitudinal Studies, Institute of Education, University of London, 2008.

18 Singer JD, Willett JB. Applied longitudinal data analysis: modeling change and event occurrence. Oxford University Press, 2003.

19 Panico L, Nazroo JY. The social and economic circumstances of mixed ethnicity children in the UK: findings from the Millennium Cohort Study. Ethnic Racial Stud 2011;34:1421-44. 